Artículo Original

\title{
Sedación consciente en tratamiento odontológico: Efectividad de dos medicaciones en niños
}

Conscious sedation in dentistry: Effectiveness of two medications in children

\section{Resumen}

La técnica de midazolam ha sido utilizada en el paciente odontopediátrico con miedo o ansiedad para lograr relajación y modificar la conducta negativa. El objetivo de este estudio fue evaluar si la hidroxicina, antihistamínico depresor del sistema nervioso central, adicionado a la técnica de midazolam vía oral proporciona niveles de sedación más adecuados.

Se seleccionaron 24 niños de 2 a 6 años de edad, ASA I, con conducta negativa (Escala de Frankl II) distribuidos al azar en dos grupos I y II; el primero recibió midazolam $0,75 \mathrm{mg} / \mathrm{kg}$ de peso y el segundo midazolam $0,75 \mathrm{mg} / \mathrm{kg}$ de peso más hidroxicina $1 \mathrm{mg} / \mathrm{kg}$ de peso. Se observó el nivel de sedación con la escala de Houpt, y se monitorizaron los signos vitales durante todo el tratamiento.

Los resultados mostraron que: en el grupo I, el 58,3 \% obtuvo niveles de sedación de Muy Bueno a Excelente, 33,3 \% Bueno a Regular y 8,3 \% pobre. Mientras que en el grupo II, el 91,7 \% obtuvo niveles de Muy Bueno a excelente, 8,3 \% Bueno a Regular y en el $0 \%$ de los casos la sedación fue Pobre ( $\mathrm{p}=0,01)$. Los valores de los signos vitales no presentaron diferencias entre los grupos y se encontraron dentro de los parámetros clínicos normales. No se mostraron efectos adversos de relevancia.

Se concluyó que la Técnica de Sedación Consciente Combinada mostró superioridad en cuanto a la efectividad, presentando ventajas estadísticamente significativas en los parámetros de movimiento y evaluación total del nivel de sedación. También, se mejoraron los efectos ansiolíticos y fueron bien tolerados por los pacientes odontopediátricos, pudiendo ser utilizada como una alternativa efectiva para el control de conductas negativas durante el tratamiento odontológico.

\section{Abstract}

The midazolam technique has been used in pediatric patients with fear or anxiety to obtain relaxation and to modify negative conduct. The objective of this study was to evaluate if hydroxyzine, an antihistaminic depressor of the central nervous system, added to midazolam technique by oral dosage provides more suitable levels of sedation.

24 children between 2 to 6 years of age, ASA I, with negative conduct (Frankl scale II) were distributed and randomized in two groups; the first group (I) received midazolam $0,75 \mathrm{mg} / \mathrm{kg}$ of weight; and the second group (II) received midazolam $0,75 \mathrm{mg} / \mathrm{kg}$ of weight plus hydroxyzine $1 \mathrm{mg} / \mathrm{kg}$ of weight. It was observed sedation level in the scale of Houpt, and vital signs were checked throughout the treatment. The results showed that: in group I, 58,3\% obtained very good to excellent, sedation levels, 33,3\% good to regular sedation levels and $8,3 \%$ poor sedation level. In the other group, $91,7 \%$ obtained very good to excellent sedation levels, $8,3 \%$ good to regular sedation levels and $0 \%$ poor sedation levels $(\mathrm{p}=0,01)$. The vital signs values didn't show differences between the groups and were within normal clinical parameters. No adverse effects of relevance were observed in any of the cases.

It was concluded that Combined Conscious Sedation Technique showed to be superior in effectiveness, showing significant statistically advantages in patient movement and in total evaluation of sedation parameters. It was also effective to provide better sedative effects and to be well tolerated by pediatric patients, being able to be used as an effective alternative for the control of negative conducts during the dental treatment.

\section{Introducción}

Los términos "miedo", "ansiedad" y "dolor" se han asociado siempre con la odontología. Los temores más frecuentes en los niños son el miedo a hablar en público y en segundo lugar el miedo a acudir al odontólogo. ${ }^{1}$ Debido a estas circunstancias, con el objeto de ofrecer al paciente pediátrico la máxima calidad, el odontólogo puede requerir un tratamiento que lo tranquilice y consiga, así, su cooperación. ${ }^{2,3}$
Ante este problema, una solución es la aplicación de técnicas farmacológicas para la disminución de la ansiedad, como lo es la sedación consciente. El midazolam es uno de los fármacos más utilizados para sedación en odontología pediátrica, cabe recalcar los diversos estudios que se han realizado de este fármaco para evaluar cual podría ser la vía de administración más eficaz y que cause menos efectos adversos al niño. ${ }^{4}$ En 1993, Karl y cols, compararon la aceptación y eficacia del midazolam en

\section{Karina del Rosario Ascanio Llaja ${ }^{1}$ María Elena Núñez Lizárraga ${ }^{2}$}

CD egresada de la Facultad de Odontología de la Universidad Nacional Mayor de San Marcos.

CD Odontopediatra Docente del Departamento Académico de Estomatología Pediátrica de la UNMSM.

\section{Correspondencia:}

Karina del Rosario Ascanio Llaja

Dirección: Jr. Manuel Mattos Bonifaz 585. Urb. San Germán. San Martín de Porres.

Teléfono: 5681491 / 995673834

E-mail: odontokari@hotmail.com

Palabras clave: Niños, Odontología, Sedación Consciente, Midazolam, Midazolam + Hidroxicina.

Keywords: Children, Dentistry, Conscious sedation, Midazolam, Midazolam + Hydroxyzine.

niños, administrado intranasalmente y por vía submucosa, y a pesar de haberse observado la misma eficacia por ambos sistemas, la administración intranasal fue la menos aceptada por la sensación "quemante" que produce el primer contacto con la mucosa nasal. ${ }^{4}$ En el 2003, Castro ${ }^{5}$ realizó una investigación, comparando la efectividad del midazolam administrado por vía oral en un grupo de niños preescolares, con la efectividad de la misma droga administrada intranasalmente a otro grupo de niños, llegando a la conclusión que 
ambas rutas de administración mostraron ser igualmente efectivas en cuanto a modificación de la conducta del niño y que los cambios fisiológicos producidos en los niños de ambos grupos se encontraron dentro de los límites normales. Por lo anterior, podemos deducir que la vía oral puede ser óptima para usar el midazolam pues muestra una buena efectividad en cuanto al manejo de conducta del niño, y se elimina esa sensación quemante que se produce al usar la vía intranasal.

Asimismo, con frecuencia resulta útil combinar varios métodos y fármacos para lograr la sedación consciente. De este modo, la proporción de un fármaco puede aumentar y potenciar el efecto de otro ${ }^{3}$.

La combinación utilizada con mayor frecuencia es la administración de fármacos orales. Es así que existen varios estudios al respecto; encontrándose en algunos de ellos resultados contradictorios como es el caso del estudio de Lima y col., ${ }^{6}$ que compararon la efectividad del uso de midazolam vía oral solo y en combinación con hidroxicina en el tratamiento de pacientes odontopediátricos, los resultados mostraron que el grupo que usó midazolam tuvo una efectividad en la sedación en un $77 \%$ de los casos y el grupo que usó midazolam-hidroxicina mostró una efectividad de solo 30,8 \%. Concluyendo que la asociación con la hidroxicina no tenía ventajas adicionales. Sin embargo, Shapira y col. ${ }^{7}$ realizaron el mismo estudio de Lima y cols., obteniendo como resultado que la combinación con hidroxicina administrada 30 minutos antes del tratamiento llevó a una sedación más segura y efectiva. Además, esta combinación fue más ventajosa en comparación con el uso del midazolam solo porque los nińos presentaron menor llanto y movimiento.

El propósito de la presente investigación fue determinar cuál técnica de sedación consciente muestra mayor efectividad, el uso de midazolam solo o su combinación con hidroxicina, valorando para ello el grado de sedación y nivel de variaciones fisiológicas que presentaron los niños que acudieron al Servicio de Odontopediatría del Centro Médico Naval (CEMENA) durante un periodo de tres meses.

\section{MATERIALES Y MÉTODOS}

El protocolo para la investigación cumplió con los principios éticos para la investigación en seres humanos (Declaración de Helsinki de la Asociación Médica Mundial) y fue aprobado por el Comité de Ética del Centro Médico Naval de la Marina de Guerra del Perú. Además, los tutores de todos los pacientes fueron informados de los posibles riesgos y benefi- cios de los procedimientos y firmaron su Consentimiento Informado.

La muestra seleccionada estuvo integrada por 24 niños (9 mujeres y 15 hombres) que acudieron al Servicio de Odontopediatría del Centro Médico Naval (CEMENA) durante los meses de noviembre y diciembre de 2006 y enero de 2007 para ser sometidos a tratamientos odontológicos bajo sedación consciente y fueron seleccionados de acuerdo a criterios de inclusión: niños cuyas edades oscilaban entre 2 y 6 años, ASA I y con conducta negativa según la Escala de Frankl (Tipo II). Distribuidos en 2 grupos: Grupo I, 12 niños a los que se les administró midazolam vía oral y Grupo II, 12 niños a los que se les administró midazolam más hidroxicina vía oral.

Los niños fueron escogidos al azar para integrar cualquiera de los grupos; previamente, fueron evaluados para ver si cumplían con los criterios de participación, siendo asimismo examinados por un médico pediatra quien determinó su condición médica saludable para realizar la sedación.

Los padres de familia fueron informados en qué consistía y por qué se iba a someter a sus niños a sedación consciente, así como las ventajas y desventajas del mismo. Además se les dio las indicaciones escritas del caso y se obtuvo el permiso del padre para que su hijo forme parte del presente estudio mediante la firma de un consentimiento informado.

Cuadro 1. Escala de Houpt para valorar el grado de sedación.

\section{Valoración del sueńo}

$4=$ Despierto y reactivo.

$3=$ Somnoliento, desorientado

$2=$ Dormido superficialmente: fácil de despertar

1 = Dormido profundamente: difícil de despertar

\section{Valoración del movimiento}

$4=$ No movimiento

$3=$ Movimiento intermitente que no afecta al tratamiento

$2=$ Movimiento continuo que afecta el tratamiento

$1=$ Movimiento violento que interrumpe, no permite el tratamiento

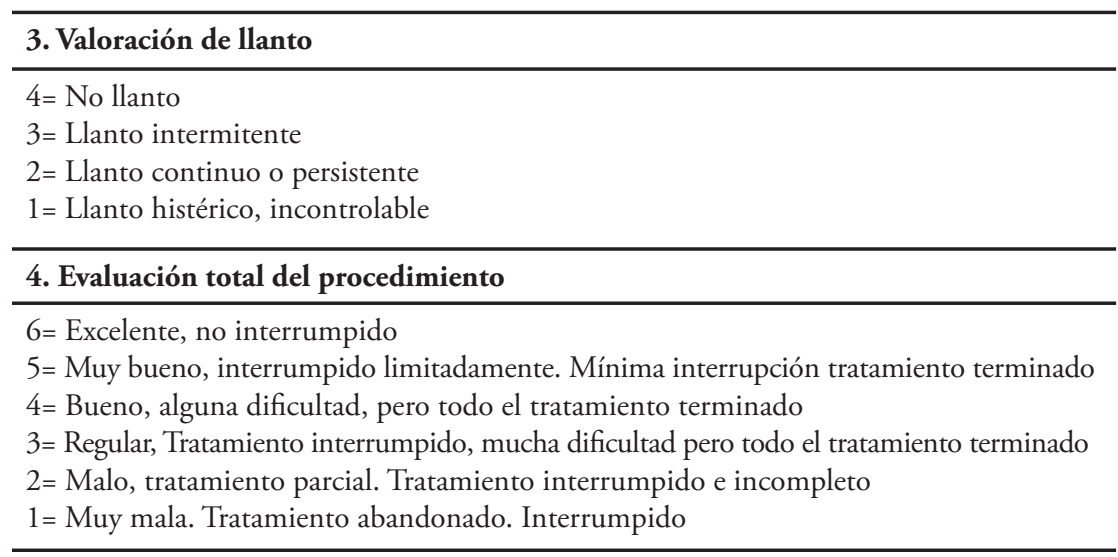

Los niños fueron citados en el primer turno porque necesitaban estar en ayunas debido a que los fármacos que se administran vía oral tienen su captación máxima con el estómago vacío.

El día de la cita se registraron los valores de las funciones vitales y el peso que sirvieron como indicadores basales y para administrar la dosis correcta según el caso (grupo I: $0,75 \mathrm{mg} / \mathrm{kg}$ de midazolam, grupo II: $0,75 \mathrm{mg} / \mathrm{kg}$ de midazolam $+1 \mathrm{mg} / \mathrm{kg}$ de hidroxicina). Las pastillas fueron molidas, diluidas y administradas al nińo, acompañado de un vehículo endulzado agradable como su jugo de preferencia.

Posterior a la ingesta de la medicación, el nińo fue conducido a la sala de descanso en compañía de sus padres por 30 minutos.

Durante este periodo, se observaron y anotaron los cambios que sufrió el niño, así como también se llevó un registro de sus funciones vitales.

Una vez que el niño estuvo listo para conducido al consultorio. Se permitió la presencia de los padres, pues este vínculo hace sentir a los niños más seguros. Durante la realización del tratamiento odontológico, se monitorizó la frecuencia cardiaca y saturación de oxígeno a través de un oxímetro de pulso y para medir la efectividad de la sedación se evaluó el comportamiento del niño a ser sometido al tratamiento dental, fue 
través de los parámetros de la escala de Houpt (Cuadro 1).

Al término del tratamiento, se dieron a los padres las indicaciones e instrucciones para el cuidado del nińo. Y se condujo al niño nuevamente a la sala de descanso para que le cambien la ropa, y permanezca durante su recuperación por un periodo de 30 minutos acompañado por sus padres y se anotaron los cambios que pudo presentar el nińo y sus funciones vitales.

Posterior a esto, el niño fue llevado a casa, haciendo hincapié a los padres de las indicaciones postoperatorias a seguir y en caso de presentarse alguna reacción adversa en el nińo, se comunique inmediatamente con la investigadora o el médico tratante.

\section{Resultados}

Los valores de la temperatura, la frecuencia respiratoria, la presión arterial, la frecuencia cardiaca, así como la saturación de oxígeno estuvieron dentro de los parámetros clínicos normales en ambos grupos de estudios.

De acuerdo a la escala de Houpt, con respecto al sueño: en el grupo I, dos pacientes se mostraron despiertos (16,7\%) y los 10 pacientes restantes se mostraron somnolientos $(83,3 \%)$, mientras que en el grupo II, sólo 1 paciente estuvo despierto $(8,3 \%)$ y los 11 restantes estuvieron somnolientos $(91,7 \%)$. En ambos casos, ninguno de los niños se durmieron ni superficialmente ni profundamente, Cuadro 2 ( $\mathrm{p}=0,755)$.

Cuadro 2. Valoración del sueño en niños sometidos a sedación consciente.

\begin{tabular}{ccccc}
\hline $\begin{array}{c}\text { Valoración } \\
\text { del } \\
\text { Sueño }\end{array}$ & Midazolam & \multicolumn{2}{c}{$\begin{array}{c}\text { Midazolam } \\
+\end{array}$} \\
\cline { 2 - 5 } & $\mathrm{n}$ & $\%$ & $\mathrm{n}$ & $\%$ \\
\hline 4 & 2 & 16,7 & 1 & 8,3 \\
3 & 10 & 83,3 & 11 & 91,7 \\
2 & 0 & 0,0 & 0 & 0,0 \\
1 & 0 & 0,0 & 0 & 0,0 \\
Total & 12 & 100 & 12 & 100 \\
\hline
\end{tabular}

$\mathrm{p}=0,755$

En lo referente al movimiento: el grupo I no presentó movimientos que interrumpieran el tratamiento en el 58,3\% de los casos, en comparación con el 91,7 \% del grupo II. Se presentaron movimientos continuos que afectaban el tratamiento en un 33,3\% en el grupo I y un $8,3 \%$ en el grupo II. Solo se registraron movimientos violentos que no permitieron la realización del tratamiento en 1 paciente del grupo I (8,3\%). Los resultados fueron estadísticamente significativos, cuadro 3 ( $\mathrm{p}=0,039)$.
Cuadro 3. Valoración del movimiento en niños sometidos a sedación consciente

\begin{tabular}{ccccc}
\hline \multirow{2}{*}{$\begin{array}{c}\text { Valoración } \\
\text { del } \\
\text { Movimiento }\end{array}$} & Midazolam & \multicolumn{2}{c}{$\begin{array}{c}\text { Midazolam } \\
+\end{array}$} \\
\cline { 2 - 5 } & $\mathrm{n}$ & $\%$ & $\mathrm{n}$ & $\%$ \\
\hline 4 & 3 & 25,0 & 8 & 66,7 \\
3 & 4 & 33,3 & 3 & 25,0 \\
2 & 4 & 33,3 & 1 & 8,3 \\
1 & 1 & 8,3 & 0 & 0,0 \\
Total & 12 & 100 & 12 & 100 \\
\hline $\mathrm{p}=0,039$ & & & &
\end{tabular}

En lo referente a la escala de llanto: los pacientes del grupo I no presentaron llanto en un $41,7 \%$, comparándolos con un $75 \%$ del grupo II. Se presentó llanto intermitente en un $50 \%$ del grupo I y un $16,7 \%$ del grupo II. Un llanto continuo se presentó en solo 1 caso de cada grupo $(8,3 \%)$, cuadro $4(\mathrm{p}=0,219)$.

Cuadro 4. Valoración del llanto en niños sometidos a sedación consciente

\begin{tabular}{ccrcc}
\hline \multirow{2}{*}{$\begin{array}{c}\text { Valoración } \\
\text { del llanto }\end{array}$} & Midazolam & \multicolumn{2}{c}{$\begin{array}{c}\text { Midazolam } \\
+\end{array}$} \\
\cline { 2 - 5 } & $\mathrm{n}$ & $\%$ & $\mathrm{n}$ & $\%$ \\
\hline 4 & 5 & 41,7 & 9 & 75,0 \\
3 & 6 & 50,0 & 2 & 16,7 \\
2 & 1 & 8,3 & 1 & 8,3 \\
1 & 0 & 0,0 & 0 & 0,0 \\
Total & 12 & 100 & 12 & 100 \\
\hline
\end{tabular}

$\mathrm{p}=0,219$

Al valorar la evaluación total del procedimiento, se demostró que solo un $25 \%$ de los pacientes a los que se les administró Midazolam se evaluó como excelente (sin interrupción), comparándolo con un 66,7 $\%$ de los que se les administró Midazolam + Hidroxicina, lo cual fue estadísticamente significativo, Cuadro 5 ( $\mathrm{p}=0,045)$.

Cuadro 5. Valoración de la Evaluación Total del Procedimiento en niños sometidos a sedación consciente

\begin{tabular}{ccrcc}
\hline $\begin{array}{c}\text { Valoración de } \\
\text { la evaluación } \\
\text { total del } \\
\text { procedimiento }\end{array}$ & Midazolam & \multicolumn{2}{c}{$\begin{array}{c}\text { Midazolam } \\
+\end{array}$} \\
\cline { 2 - 5 } & $\mathrm{n}$ & $\%$ & $\mathrm{n}$ & $\%$ \\
\hline 6 & 3 & 25,0 & 8 & 66,7 \\
5 & 4 & 33,3 & 3 & 25,0 \\
4 & 2 & 16,7 & 0 & 0,0 \\
3 & 2 & 16,7 & 1 & 8,3 \\
2 & 1 & 8,3 & 0 & 0,0 \\
1 & 0 & 0,0 & 0 & 0,0 \\
Total & 12 & 100 & 12 & 100 \\
\hline $\mathrm{p}=0,045$ & & & &
\end{tabular}

$\mathrm{p}=0,045$
Para simplificar la Evaluación del Nivel de Sedación alcanzado según la escala de Houpt, se agrupó los 6 niveles en tres categorías: pobre, bueno y excelente, al igual que en el estudio de Cortés y col. ${ }^{8}$ Demostrándose que el 91,7 \% de los pacientes a los que se les administró la técnica combinada, se evaluó como Muy bueno-excelente (mínima interrupción), comparándolo con un $58,3 \%$ de los que se les administró Midazolam. Asimismo, los tratamientos se evaluaron como Bueno - Regular (cierta interrupción, pero tratamiento culminado) en un $33,3 \%$ en el grupo I y un $8,3 \%$ en el grupo II. Se evaluó como pobre (tratamiento NO terminado), solo 1 caso en el grupo I (8,3\%). Se observó finalmente, que el grupo en el que se utilizó la técnica combinada (Midazolam + Hidroxicina) presentó niveles de sedación más favorables durante el tratamiento, y por ende fue más efectiva (Fig.1).

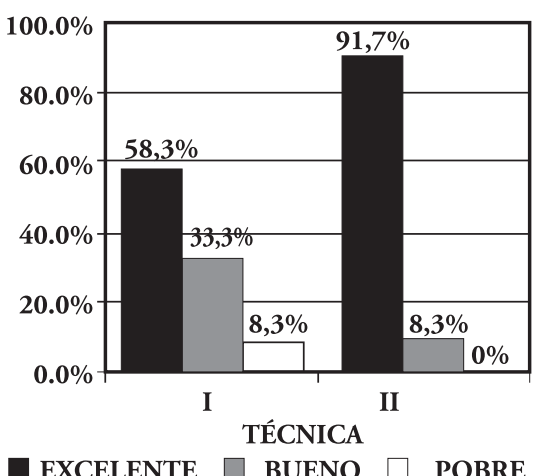

Fig 1. Efectividad obtenida con ambas técnicas de sedación consciente en niños sometidos a tratamientos odontológicos en el Servicio de Odontopediatría del Centro Médico Naval.

En ninguno de los casos se mostraron reacciones adversas de relevancia, pero cabe resaltar tres manifestaciones que se presentaron en solo 5 pacientes de la muestra total: llanto postratamiento en 2 nińos del grupo I y 1 del grupo II; y esfínteres e hipo no controlado en solo 1 caso en cada grupo.

\section{Discusión}

A nivel internacional, se han realizado múltiples estudios sobre combinaciones de medicamentos que reportan resultados alentadores en la búsqueda de un esquema farmacológico seguro y eficiente. En nuestro país, existen pocos reportes al respecto.

En esta investigación, aunque la distribución de grupos fue hecha al azar, encontramos homogeneidad en cuanto al 
género y edad de ambos, lo que nos habla de que no existe diferencia significativa para indicar una sedación tomando en cuenta la edad o el sexo del paciente. Hartgraves y Primosch ${ }^{9}$ encontraron los mismos resultados.

En cuanto al comienzo de los efectos sedantes, para los que recibieron midazolam, se dieron a los 30 minutos de su administración. Coincidiendo con ello, Castro $^{5}$ en su estudio de midazolam administrado por vía oral en diez pacientes, que a los 30 minutos de haber administrado la dosis de $0,5 \mathrm{mg} / \mathrm{kg}$ de peso, se comenzaban a apreciar los efectos del sedante. El grupo que recibió la técnica combinada también mostró un periodo de latencia similar al que recibió midazolam.

Con respecto a la administración de los medicamentos, se pudo observar una buena aceptación del paciente. Esto se debió a que el medicamento fue mezclado con un jugo de sabor agradable para el niño, lo cual trata de contrarrestar el mal sabor de los fármacos. En estudios previos, Hartgraves contrarrestó el mal sabor mezclando el midazolam con 25 $\mathrm{mg}$ de hidroxicina azucarada, con lo cual se logró la aceptación del paciente. ${ }^{9}$ Igualmente Chávez y cols. ${ }^{10}$ utilizaron como vía de administración jugo de manzana $(5 \mathrm{ml})$ mezclándolo con midazolam $(0,75 \mathrm{mg} / \mathrm{kg}$ de peso) para darle mejor sabor y obteniéndose la aceptación por los paciente pediátricos.

Los resultados obtenidos en cuanto a los signos vitales confirmaron la seguridad de ambos esquemas de sedación. Los valores de frecuencia cardiaca, frecuencia respiratoria, presión arterial, temperatura y saturación de oxígeno durante toda la evaluación del estudio, tanto en los pacientes sedados con midazolam y la técnica combinada se mantuvieron dentro de los parámetros clínicos normales. Solo la frecuencia cardiaca y la presión arterial sistólica mostraron diferencias estadísticamente significativas entre ambos grupos, pues tendieron a aumentar y luego se normalizaron pero estos valores oscilaron dentro de los límites normales.

Nuestros hallazgos respecto a la estabilidad de los signos vitales confirman las conclusiones de estudios anteriores como el de Juárez y col., ${ }^{11}$ en el que no se encontraron alteraciones fisiológicas negativas en ninguno de sus tres grupos de estudio (midazolam más hidroxicina, midazolam y placebo).

En el estudio de Wilson y cols. ${ }^{12}$ encontraron diferencias estadísticamente signi- ficativas entre los regímenes de drogas en cuanto la frecuencia cardiaca y la presión arterial media; sin embargo, todas las variables fisiológicas estuvieron dentro de los límites normales para niños.

No hubieron reportes de náuseas, vómitos u otros efectos adversos de relevancia ni durante ni después de haber concluido la sedación. Lo que nos confirma la seguridad de ambos esquemas de sedación. De igual manera, Lima y cols. ${ }^{6}$ en su ensayo clínico controlado, cruzado y doble ciego para comparar el efecto sedativo en odontopediatría de la administración oral de midazolam, asociado o no a hidroxicina, no observaron efectos adversos de importancia en ninguno de sus grupos de estudio.

La evaluación del grado de sedación a través de la modificación de la conducta bajo los efectos sedantes se realizó utilizando la escala de clasificación de conducta diseñada por Houpt. La cual evalúa cuatro parámetros: llanto, sueño, movimiento y evaluación total del procedimiento, encontrándose que no hubo diferencias estadísticamente significativas con llanto y somnolencia; sin embargo, en lo referente al movimiento y evaluación total del procedimiento, sí hubo diferencias significativas, observándose menos movimiento en los niños sedados con la técnica combinada.

En el presente estudio el grupo que recibió además de midazolam, la hidroxicina, presentó mayor cooperación con el tratamiento dental, el $75 \%$ de los niños en estudio, no presentaron llanto contra un $41,7 \%$ del esquema sin hidroxicina $(\mathrm{p}=0,219)$. Al valorar el movimiento, encontramos que al usar la técnica combinada durante el procedimiento dental, el movimiento fue escaso; mientras que al usar solamente midazolam, el movimiento llegó inclusive a afectar la realización del procedimiento. Durante el tratamiento, el valor del sueño fue homogéneo en ambos grupos.

Sin embargo, existen diferencias con respecto a la evaluación general, el grupo que recibió el esquema con hidroxicina presentó en el 91,7\% de los casos, niveles de sedación en los rangos de muy bueno a excelente, 8,3\% de regular a bueno y $0 \%$ pobre, mientras que en el otro grupo, sólo el 58,3\% obtuvo niveles de bueno a excelente, el 33,3\% de regular a bueno y en el 8,3\% de los casos, la sedación fue pobre.

Basándonos en los resultados anteriores, se puede considerar que la hidroxicina potencia el efecto del midazolam. Por lo cual, la técnica que se basa en la asociación midazolam - hidroxicina es más efectiva por los beneficios que brinda en el ámbito odontopediátrico.

Las observaciones clínicas realizadas en el presente estudio confirman los resultados de investigaciones reportadas en la literatura odontopediátrica, como la de Shapira y col.7 Que al comparar la efectividad del midazolam solo y en combinación con hidroxicina en la sedación para tratamientos de pacientes odontopediátricos, hallaron que el grupo que recibió midazolam mostró más niños que exhibieron movimientos; asimismo más niños lloraron en este grupo, mientras que el grupo que recibió midazolam más hidroxicina presentó más niños somnolientos. Concluyendo que la combinación de hidroxicina con midazolam administrado 30 minutos antes del tratamiento resultó seguro y más efectivo en la sedación para el tratamiento de niños.

\section{Conclusiones}

- Los resultados obtenidos muestran que la técnica combinada (midazolam + hidroxicina) fue más efectiva (91,7\%).

- Ambas técnicas de sedación consciente son seguras pues los cambios fisiológicos observados están dentro de los límites normales sin mostrar diferencias estadísticamente significativas.

- Ninguna de las dos técnicas presentaron algún tipo de reacciones adversas de relevancia en el presente trabajo de investigación.

- La técnica combinada (midazolam más hidroxicina) puede ser más ventajosa porque los pacientes presentan menor llanto y movimiento.

- No se requirió suplemento de oxígeno y no se presentaron complicaciones.

\section{Referencias bibliográficas}

1. Rojano A, Pizano MA, Banderas JA. Dosis efectiva de midazolam para sedación consciente en estomatología pediátrica. Rev ADM 2004; 61(4):130-6

2. Castillo DD, Gutiérrez A y Ruiz LO. Sedación y analgesia en urgencias pediátricas. Rev Cubana de Med Gen Integr 2003; 19(2)

3. McDonald RE, Avery DR. Odontología Pediátrica y del adolescente. $\sigma^{a}$ ed. España: Edit. Mosby/ Doyma Libros, 1995: 293-317. 
4. Díaz ME. Sedación consciente en odontopediatría. El uso de midazolam por vía intranasal, ¿una alternativa eficaz. Visión Dental Rev Estomatol Peruana-Sept 2006.

5. Castro ES. Efectividad del midazolam en sedación consciente de niños preescolares en la clínica dental UPCH, vía oral vs. vía intranasal.[ Tesis para optar el título de Cirujano Dentista ]. Lima: Universidad Peruana Cayetano Heredia; 2003.

6. Lima AR, Da Costa LR, Da Costa PS. A randomized, controlled, crossover trial of oral midazolam and hydroxyzine for pediatric dental sedation. Pesqui Odontol Bras 2003; 17(3):206-11
7. Shapira J, Kupietzky A, Kadari A, Fuks AB, Holan G. Comparison of oral midazolam with and without hydroxyzine in the sedation of pediatric dental patients. Pediatr Dent 2004; 26(6):492-6.

8. Cortés A, Juárez LA, Ramírez G. Evaluación clínica de óxido nitroso- midazolam-acetaminofén para modificar la conducta en pacientes odontopediátricos. Rev ADM 2002; 59(2):45-9.

9. Hartgraves PM, Primosch RE. An evaluation of oral and nasal midazolam for pediatric dental sedation. J Dent Child 1994; 6(3):175-80.

10. Chávez EJ, Cortés E, Hinojosa A, Araiza MA. Estudio comparativo de la premedicación con mida- zolam y ketamina por vía oral en pacientes pediátricos sometidos a tratamiento de cirugía maxilofacial. Rev Odontol Mexicana 2005; 9(3):131-6.

11. Juárez LA, Saavedra M, Ramírez G. Estudio comparativo entre dos esquemas de sedación en pacientes odontopediátricos. Bol méd Hosp Infant Méx-Ago 1998; 55(8):443-51.

12. Wilson S, Easton J, Lamb K, Orchardson R, Cassamassino P. A retrospective study of chloral hydrate, meperidine, hydroxyzine, and midazolam regimens used to sedate children for dental care. Pediatr Dent 2000; 22(2):107-12.

Fecha de recepción: 18 de diciembre 2009

Fecha de aprobación: 5 de enero 2010 